\title{
Derivation of Jump Conditions in Multiphase Incompressible Flows with Singular Forces
}

\author{
Caesar O. HARAHAP and Hideaki MIURA ${ }^{1)}$ \\ The Graduate University for Advanced Studies (SOKENDAI), Toki 509-5292, Japan \\ ${ }^{1)}$ National Institute for Fusion Science, Toki 509-5292, Japan
}

(Received 21 November 2007 / Accepted 10 March 2008)

\begin{abstract}
For conducting numerical simulations of plasma dynamics consisting of multiple phases, a new immersed interface method (IIM) scheme to solve multiphase flows with different viscosities and densities is being developed. The jump conditions for velocity, pressure, and their derivatives necessary for the finite difference approximations in the IIM are derived. The derivation results in sets of coupled equations that can be solved numerically by an iterative method.
\end{abstract}

(c) 2008 The Japan Society of Plasma Science and Nuclear Fusion Research

Keywords: discontinuity, jump condition, immersed interface method

DOI: $10.1585 /$ pfr.3.S1052

\section{Introduction}

Clarifying key physics of complex behaviors of a hot plasma in a magnetic confinement device such as Large Helical Device (LHD) is essential for understanding experimental results. For this purpose, various kinds of simulation studies such as magnetohydrodynamics (MHD), twofluid, Vlasov, and gyrokinetic simulations have been conducted (see, for example, Refs. [1-3]). The MHD simulation class is the simplest among them in the sense that the system of equations consists of a relatively few number of equations, and the number of dimensions of the independent variable space is only three. Nevertheless, there still remain many difficulties mimicking the real device geometry in detail. One of those difficulties is in connecting the hot plasma region and the vacuum region, which is complicated because the governing equations of these regions can be different from each other.

In MHD simulations, the vacuum region is often described by MHD equations with very low pressure and/or mass density or simply omitted from the simulation by imposing boundary conditions on the outermost magnetic surface. For the purpose of studying the effects of plasma deformations around the hot plasma boundary or to study peripheral regions and hot plasma core simultaneously, the former approach is preferable. In the former simulations, very large resistivity and/or viscosity are assumed to the low pressure region. However, jumps of physical variables such as mass density, pressure, and temperature often cause numerical oscillations. Although such oscillations may be avoided by adopting numerical techniques such as the Godunov/CIP or TVD schemes (see, for example, Refs. [4,5]), the computation program often becomes complex and the numerical accuracy can become ambiguous.

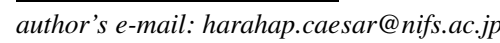

The immersed interface method (IIM) is a class of numerical schemes for partial differential equations involving interfaces and irregular domains. It is a sharp interface method that can accurately capture discontinuities in the solution and the flux. It has been applied to various problems including the simulation of electromigration of voids, Stefan problems, and incompressible flows with moving interfaces [6]. The basic properties of the IIM are as follows [6]: (1) prior knowledge of jump conditions either from physical reasoning or derived from the governing equations and (2) modifications of finite difference approximations at grid points near the interface, based on the jump conditions.

In the present paper, a moving surface separating two regions of a fluid or two fluids that the fluid(s) cannot flow across is called a singular surface. Singular forces such as surface tension or membrane elastic tension act on this singular surface affecting the dynamics of the fluid-surface system. A cage of the magnetic field lines separating the hot plasma core from the peripheral regions can be modeled as a singular surface.

Recently, Xu and Wang [7-9] developed an IIM related scheme to simulate the motion of an incompressible neutral fluid separated into two regions by a singular surface. The jump conditions in such a system were derived analytically [7]. They are then used [8,9] in finite difference simulations based on the generalized Taylor expansion [7].

While the scheme of $\mathrm{Xu}$ and Wang can be applied to many problems, it still has limitations, which may be essential for our aim to apply the scheme to hot plasma problems. One of them is that their scheme is not applicable to flows with variable density and/or viscosity, that is, multiphase flows. In this paper, we derive jump conditions in a multiphase incompressible flow where the viscosity and 
the mass density are discontinuous across the singular surface. The results can be used to develop an IIM scheme for such multiphase flows. By extending it to multiphase flows, we take one important step to make the IIM applicable to fusion plasma simulations.

\section{Governing Equations and IIM Dis- cretization}

A schematic view of the system we are concerned with is given in Fig. 1 . The fluid density $\rho$ and the viscosity $\mu$ are piecewise constant in $\Omega^{+}$and $\Omega^{-}$. We consider the situation that a singular force is applied on the closed surface $S$. It is emphasized that the separation of the two fluid phases is sustained by this singular force.

Using the index notation and the summation convention, the incompressible Navier-Stokes equations subject to a singular force are

$\rho\left(\frac{\partial u^{i}}{\partial t}+u^{j} \frac{\partial u^{i}}{\partial x^{j}}\right)=-\frac{\partial p}{\partial x^{i}}+\frac{\partial}{\partial x^{j}}\left(\mu \frac{\partial u^{i}}{\partial x^{j}}+\mu \frac{\partial u^{j}}{\partial x^{i}}\right)+F^{i}$,

$\frac{\partial u^{i}}{\partial x^{i}}=0$

where $x^{i}(i=1,2,3)$ is in Cartesian coordinates, $t$ is time, $u^{i}$ is velocity, $p$ is pressure, and $F^{i}$ is the singular force. The singular force is given by

$F^{i}=\int_{S} f^{i}\left(\alpha^{1}, \alpha^{2}, t\right) \delta\left(\boldsymbol{x}-\boldsymbol{X}\left(\alpha^{1}, \alpha^{2}, t\right)\right) \mathrm{d} \alpha^{1} \mathrm{~d} \alpha^{2}$,

where $\boldsymbol{X}\left(\alpha^{1}, \alpha^{2}, t\right)$ denotes the coordinates of a point on the singular surface, $\delta\left(\boldsymbol{x}-\boldsymbol{X}\left(\alpha^{1}, \alpha^{2}, t\right)\right)$ is a three-

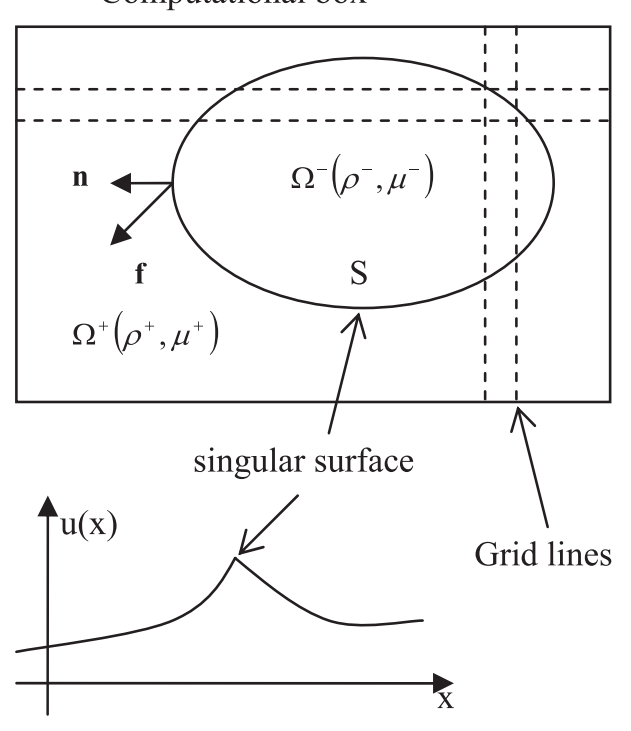

Fig. 1 Schematic view of two fluids separated by a singular surface. dimensional Dirac delta function, $f^{i}\left(\alpha^{1}, \alpha^{2}, t\right)$ is the surface force density, and $\left(\alpha^{1}, \alpha^{2}\right)$ are Lagrangian parameters of points on the surface at a reference time (see Fig. 2). The singular surface moves with the local fluid velocity

$$
\frac{\partial X^{i}\left(\alpha^{1}, \alpha^{2}, t\right)}{\partial t}=u^{i}\left(\boldsymbol{X}\left(\alpha^{1}, \alpha^{2}, t\right), t\right)
$$

Taking the divergence of eq. (1), we obtain a Poisson equation for pressure.

$$
\begin{aligned}
\frac{\partial^{2} p}{\partial x^{i} \partial x^{i}}= & \frac{\partial F^{i}}{\partial x^{i}}+\frac{\partial}{\partial x^{i}}\left\{\frac{\partial}{\partial x^{j}}\left(\mu \frac{\partial u^{i}}{\partial x^{j}}\right)\right\} \\
& +\frac{\partial}{\partial x^{i}}\left\{\frac{\partial}{\partial x^{j}}\left(\mu \frac{\partial u^{j}}{\partial x^{i}}\right)\right\}-\frac{\partial}{\partial x^{i}}\left(\rho \frac{\mathrm{D} u^{i}}{\mathrm{D} t}\right),
\end{aligned}
$$

where $\frac{\mathrm{D}}{\mathrm{D} t}=\frac{\partial}{\partial t}+u^{j} \frac{\partial}{\partial x^{j}}$.

These governing equations can be solved numerically on a fixed Cartesian mesh using the IIM finite difference discretization derived systematically by $\mathrm{Xu}$ and Wang [7]. Here, we describe the IIM discretization briefly. The reader is referred to [7] for the derivation.

Consider the function $g(x)$ in Fig. 2. It is smooth except at the discontinuity points $\xi$ and $\eta$ (note that $x_{i-1}<$ $\xi<x_{i}$ and $\left.x_{i}<\eta<x_{i+1}\right)$. In this case, the first and second derivatives at $x_{i}$ are given by

$$
\begin{aligned}
\frac{\mathrm{d} g\left(x_{i}\right)}{\mathrm{d} x}= & \frac{g\left(x_{i+1}\right)-g\left(x_{i-1}\right)}{2 h} \\
& -\frac{1}{2 h}\left[\sum_{n=0}^{2} \frac{\left[g^{(n)}(\xi)\right]}{n !}\left(x_{i-1}-\xi\right)^{n}\right. \\
& \left.+\sum_{n=0}^{2} \frac{\left[g^{(n)}(\eta)\right]}{n !}\left(x_{i+1}-\eta\right)^{n}\right)+O\left(h^{2}\right),
\end{aligned}
$$

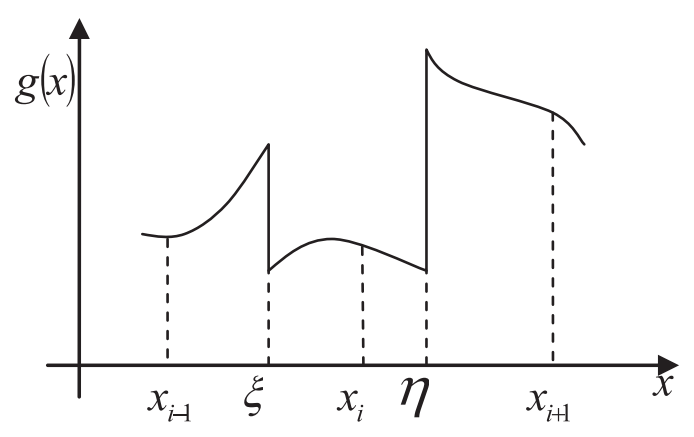

Fig. $2 g(x)$ is discontinuous at $\xi$ and $\eta$. Ordinary finite difference formulae do not hold at $x_{i}$. 


$$
\begin{aligned}
\frac{\mathrm{d}^{2} g\left(x_{i}\right)}{\mathrm{d} x}= & \frac{g\left(x_{i+1}\right)-2 g\left(x_{i}\right)+g\left(x_{i-1}\right)}{h^{2}} \\
& +\frac{1}{h^{2}}\left[\sum_{n=0}^{3} \frac{\left[g^{(n)}(\xi)\right]}{n !}\left(x_{i-1}-\xi\right)^{n}\right. \\
& \left.-\sum_{n=0}^{3} \frac{\left[g^{(n)}(\eta)\right]}{n !}\left(x_{i+1}-\eta\right)^{n}\right)+O\left(h^{2}\right),
\end{aligned}
$$

where $h=x_{i}-x_{i-1}=x_{i+1}-x_{i}$, and $[*]=*^{+}-*^{-}$denotes the jump of a quantity across a discontinuity (the jump condition for a quantity).

The finite differences in Eqs. (6) and (7) can be used to approximate the spatial derivatives appearing in the governing equations, only if the jump conditions for the velocity, pressure, and their first and second order spatial derivatives are known (the accuracy will be first order for the second order derivatives). Therefore, the derivation of these jump conditions is a prerequisite for solving the governing equations by the IIM.

\section{Principal Jump Conditions}

To prepare for the derivation of the jump conditions, we introduce some notations related to the geometry of the singular surface. Differentiating $\boldsymbol{X}$, two tangent vectors along the Lagrangian parameter lines are obtained.

$$
\boldsymbol{\tau}_{i}=\frac{\partial \boldsymbol{X}}{\partial \alpha^{i}} \quad(i=1,2) .
$$

The three components of $\tau_{i}$ are denoted by $\left(\tau_{i}^{1}, \tau_{i}^{2}, \tau_{i}^{3}\right)$. The unit vector $\boldsymbol{n}$ normal to the surface is given by

$$
\begin{aligned}
& \boldsymbol{n}=\frac{1}{J}\left(\boldsymbol{\tau}_{1} \times \boldsymbol{\tau}_{2}\right), \\
& J=\left|\boldsymbol{\tau}_{1} \times \boldsymbol{\tau}_{2}\right| .
\end{aligned}
$$

We also introduce three mutually orthogonal unit vectors as $\boldsymbol{\tau}=\tau_{1} /\left|\tau_{1}\right|, \quad \boldsymbol{n}, \quad \boldsymbol{b}=\boldsymbol{n} \times \boldsymbol{\tau}$ (see Fig. 3 ). Note that these three vectors satisfy the following identity.

$$
n^{i} n^{k}=\delta^{i k}-\tau^{i} \tau^{k}-b^{i} b^{k}
$$

We define a coordinate transformation

$$
x^{i}=x^{i}\left(\alpha^{1}, \alpha^{2}, \alpha^{3} ; t\right), \quad \alpha^{i}=\alpha^{i}\left(x^{1}, x^{2}, x^{3} ; t\right),
$$

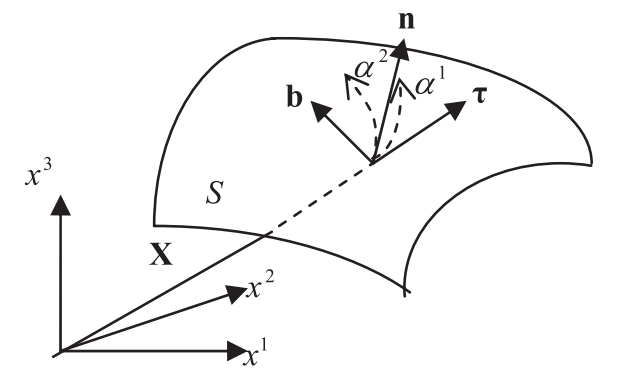

Fig. 3 Cartesian coordinates, curvilinear coordinates, unit normal and tangential vectors. where $\alpha^{3}$ is a new coordinate with

$$
\boldsymbol{x}\left(\alpha^{1}, \alpha^{2}, \alpha^{3}=0 ; t\right)=\boldsymbol{X}\left(\alpha^{1}, \alpha^{2}, t\right),
$$

and chosen to satisfy

$$
\frac{\partial \boldsymbol{x}\left(\alpha^{1}, \alpha^{2}, \alpha^{3}=0 ; t\right)}{\partial \alpha^{3}}=\boldsymbol{n},
$$

implying

$$
\nabla \alpha^{3}=n
$$

Let $\tilde{f}^{i}$ be a contravariant component of the surface force density in the curvilinear coordinate system. It is related to $f^{i}$ by

$$
\tilde{f}^{i}=\frac{\partial \alpha^{i}}{\partial x^{j}} f^{j}
$$

The jump of a physical quantity $\psi$ across the singular surface is denoted as

$$
\begin{aligned}
{[\psi]\left(\alpha^{1}, \alpha^{2}, t\right)=} & (\psi)^{+}\left(\boldsymbol{X}\left(\alpha^{1}, \alpha^{2}, t\right), t\right) \\
& -(\psi)^{-}\left(\boldsymbol{X}\left(\alpha^{1}, \alpha^{2}, t\right), t\right),
\end{aligned}
$$

where the superscript $\left(^{+}\right)$denotes the $\Omega^{+}$side, while $\left(^{-}\right)$denotes the $\Omega^{-}$side.

The principal jump conditions are derived as follows. First, no slip condition on the singular surface implies the continuity of the velocity across the surface.

$$
\left[u^{i}\right]=0 \text {. }
$$

Differentiating this equation with respect to $t$ yields

$$
\left[\frac{\partial u^{i}}{\partial t}\right]+u^{j}\left[\frac{\partial u^{i}}{\partial x^{j}}\right]=0,
$$

which states that the acceleration is also continuous across the surface.

Take an infinitesimal area $\delta S=J \delta \alpha^{1} \delta \alpha^{2}$ on the singular surface $S$. Translate $\delta S$ in the directions of $\boldsymbol{n}$ and $-\boldsymbol{n}$ by $\varepsilon / 2$, and denote the swept region as $\delta V$. Integrate Eq. (1) over $\delta V$ and take the limit $\varepsilon \rightarrow 0$.

$$
\begin{aligned}
\lim _{\varepsilon \rightarrow 0} & \int_{\delta V} \rho\left(\frac{\partial u^{i}}{\partial t}+u^{j} \frac{\partial u^{i}}{\partial x^{j}}\right) \mathrm{d} V=\lim _{\varepsilon \rightarrow 0} \int_{\delta V} F^{i} \mathrm{~d} V \\
& +\lim _{\varepsilon \rightarrow 0} \int_{\delta V} \frac{\partial}{\partial x^{j}}\left\{-p \delta^{i j}+\mu\left(\frac{\partial u^{i}}{\partial x^{j}}+\frac{\partial u^{j}}{\partial x^{i}}\right)\right\} \mathrm{d} V .
\end{aligned}
$$

From this equation, we obtain the following jump conditions (see Appendix A for the detailed calculation).

$$
\begin{gathered}
{[p]=\frac{f^{i} n^{i}}{J}+2\left[\mu \frac{\partial u^{i}}{\partial x^{j}}\right] n^{j} n^{i},} \\
{\left[\mu \frac{\partial u^{i}}{\partial x^{j}}\right] n^{j}+\left[\mu \frac{\partial u^{j}}{\partial x^{i}}\right] n^{j}} \\
\frac{f^{k} n^{k} n^{i}-f^{i}}{J}+2\left[\mu \frac{\partial u^{k}}{\partial x^{j}}\right] n^{j} n^{k} n^{i} .
\end{gathered}
$$


Next, we derive the jump condition for the normal derivative of pressure. Take control volume $V_{s}$, which is a layer with thickness $\varepsilon$ containing the singular surface $S$. Multiply Eq. (5) by an arbitrary smooth function $\phi(x)$, integrate over $V_{s}$, and take the limit $\varepsilon \rightarrow 0$.

$$
\begin{aligned}
\lim _{\varepsilon \rightarrow 0} & \int_{V_{s}} \phi \frac{\partial^{2} p}{\partial x^{i} \partial x^{i}} \mathrm{~d} V=\lim _{\varepsilon \rightarrow 0} \int_{V_{s}} \phi \frac{\partial F^{i}}{\partial x^{i}} \mathrm{~d} V \\
& +\lim _{\varepsilon \rightarrow 0} \int_{V_{s}} \phi \frac{\partial}{\partial x^{i}}\left\{\frac{\partial}{\partial x^{j}}\left(\mu \frac{\partial u^{i}}{\partial x^{j}}\right)+\frac{\partial}{\partial x^{j}}\left(\mu \frac{\partial u^{j}}{\partial x^{i}}\right)\right\} \mathrm{d} V \\
& -\lim _{\varepsilon \rightarrow 0} \int_{V_{s}} \phi \frac{\partial}{\partial x^{i}}\left(\rho \frac{D u^{i}}{D t}\right) \mathrm{d} V .
\end{aligned}
$$

By applying the generalized Gauss theorem [7], the coordinate transformation, and some other manipulations, the following jump condition can be derived from this equation (see Appendix B for the detailed calculation).

$$
\begin{gathered}
{\left[\frac{\partial p}{\partial x^{i}}\right] n^{i}=\frac{1}{J}\left(\frac{\partial \tilde{f}^{1}}{\partial \alpha^{1}}+\frac{\partial \tilde{f}^{2}}{\partial \alpha^{2}}\right)} \\
+2 \frac{\partial}{\partial \tau}\left(\left[\mu \frac{\partial u^{i}}{\partial x^{j}}\right]\right) n^{i} \tau^{j}+2 \frac{\partial}{\partial b}\left(\left[\mu \frac{\partial u^{i}}{\partial x^{j}}\right]\right) n^{i} b^{j} \\
+\left[\mu \frac{\partial u^{i}}{\partial x^{j}}\right]\left(\frac{\partial}{\partial \tau}\left(n^{j} \tau^{i}+n^{i} \tau^{j}\right)\right. \\
\left.+\frac{\partial}{\partial b}\left(n^{j} b^{i}+n^{i} b^{j}\right)\right)-[\rho] \frac{\mathrm{D} u^{i}}{\mathrm{D} t} n^{i} .
\end{gathered}
$$

\section{Scaled Velocity and Augmented Variables}

We transform the velocity into a scaled velocity as

$$
v^{i}=\mu u^{i}
$$

and introduce augmented variables

$$
q^{i}\left(\alpha^{1}, \alpha^{2}, t\right)=\left[v^{i}\right]
$$

defined only on the singular surface. Note that using the scaled velocity for $x \notin S$, Eqs. (1) and (5) become

$$
\begin{aligned}
& \frac{\rho}{\mu} \frac{\partial v^{i}}{\partial t}+\frac{\rho}{\mu^{2}} v^{j} \frac{\partial v^{i}}{\partial x^{j}}=-\frac{\partial p}{\partial x^{i}}+\frac{\partial^{2} v^{i}}{\partial x^{j} \partial x^{j}}, \\
& \frac{\partial^{2} p}{\partial x^{i} \partial x^{i}}=-\frac{\rho}{\mu^{2}} \frac{\partial v^{j}}{\partial x^{i}} \frac{\partial v^{i}}{\partial x^{j}} .
\end{aligned}
$$

We emphasize here that the transformation into the scaled velocity and the introduction of the augmented variables will simplify both the coupled equations for the jump conditions and the numerical solution of the multiphase flow.

\section{Jump Conditions of Spatial Deriva- tives}

In this section, we derive jump conditions for derivatives of the scaled velocity and pressure. First, differentiating Eq. (26) with respect to $\alpha^{m}(m=1,2)$ yields

$$
\left[\frac{\partial v^{i}}{\partial x^{j}}\right] \tau_{m}^{j}=\frac{\partial q^{i}}{\partial \alpha^{m}} .
$$

Using Eq. (11), Eq. (22) becomes

$$
\begin{aligned}
{\left[\frac{\partial v^{i}}{\partial x^{j}}\right] n^{j}+\left[\frac{\partial v^{j}}{\partial x^{i}}\right] n^{j}=} & \frac{f_{n} n^{i}-f^{i}}{J} \\
& -2 \frac{\partial q^{j}}{\partial \tau} \tau^{j} n^{i}-2 \frac{\partial q^{j}}{\partial b} b^{j} n^{i},
\end{aligned}
$$

where $f_{n}=f^{i} n^{i}$. Eqs. (29) and (30) form a set of equations that can be written explicitly as

$$
\begin{aligned}
& \left(\begin{array}{ccccccccc}
\tau_{1}^{1} & \tau_{1}^{2} & \tau_{1}^{3} & 0 & 0 & 0 & 0 & 0 & 0 \\
\tau_{2}^{1} & \tau_{2}^{2} & \tau_{2}^{3} & 0 & 0 & 0 & 0 & 0 & 0 \\
2 n^{1} & n^{2} & n^{3} & n^{2} & 0 & 0 & n^{3} & 0 & 0 \\
0 & 0 & 0 & \tau_{1}^{1} & \tau_{1}^{2} & \tau_{1}^{3} & 0 & 0 & 0 \\
0 & 0 & 0 & \tau_{2}^{1} & \tau_{2}^{2} & \tau_{2}^{3} & 0 & 0 & 0 \\
0 & n^{1} & 0 & n^{1} & 2 n^{2} & n^{3} & 0 & n^{3} & 0 \\
0 & 0 & 0 & 0 & 0 & 0 & \tau_{1}^{1} & \tau_{1}^{2} & \tau_{1}^{3} \\
0 & 0 & 0 & 0 & 0 & 0 & \tau_{2}^{1} & \tau_{2}^{2} & \tau_{2}^{3} \\
0 & 0 & n^{1} & 0 & 0 & n^{2} & n^{1} & n^{2} & 2 n^{3}
\end{array}\right) \\
& \times\left(\begin{array}{l}
{\left[\partial v^{1} / \partial x^{1}\right]} \\
{\left[\partial v^{1} / \partial x^{2}\right]} \\
{\left[\partial v^{1} / \partial x^{3}\right]} \\
{\left[\partial v^{2} / \partial x^{1}\right]} \\
{\left[\partial v^{2} / \partial x^{2}\right]} \\
{\left[\partial v^{2} / \partial x^{3}\right]} \\
{\left[\partial v^{3} / \partial x^{1}\right]} \\
{\left[\partial v^{3} / \partial x^{2}\right]} \\
{\left[\partial v^{3} / \partial x^{3}\right]}
\end{array}\right)=\left(\begin{array}{c}
\frac{\partial q^{1}}{\partial \alpha^{1}} \\
\frac{\partial q^{1}}{\partial \alpha^{2}} \\
R_{v}^{1} \\
\frac{\partial q^{2}}{\partial \alpha^{1}} \\
\frac{\partial q^{2}}{\partial \alpha^{2}} \\
R_{v}^{2} \\
\frac{\partial q^{3}}{\partial \alpha^{1}} \\
\frac{\partial q^{3}}{\partial \alpha^{2}} \\
R_{v}^{3}
\end{array}\right)
\end{aligned}
$$

where

$$
R_{v}^{i}=\frac{f_{n} n^{i}-f^{i}}{J}-2 \frac{\partial q^{j}}{\partial \tau} \tau^{j} n^{i}-2 \frac{\partial q^{j}}{\partial b} b^{j} n^{i}
$$

Next, we differentiate Eq. (21) with respect to $\alpha^{m}(m=1,2)$ to obtain

$$
\left[\frac{\partial p}{\partial x^{i}}\right] \tau_{m}^{i}=\frac{\partial}{\partial \alpha^{m}}\left(\frac{f_{n}}{J}+2\left[\frac{\partial v^{i}}{\partial x^{j}}\right] n^{j} n^{i}\right) .
$$

This equation and Eq. (24) form the following set of equations.

$$
\left(\begin{array}{ccc}
\tau_{1}^{1} & \tau_{1}^{2} & \tau_{1}^{3} \\
\tau_{2}^{1} & \tau_{2}^{2} & \tau_{2}^{3} \\
n^{1} & n^{2} & n^{3}
\end{array}\right) \times\left(\begin{array}{l}
{\left[\frac{\partial p}{\partial x^{1}}\right]} \\
{\left[\frac{\partial p}{\partial x^{2}}\right]} \\
{\left[\frac{\partial p}{\partial x^{3}}\right]}
\end{array}\right)=\left(\begin{array}{c}
\frac{\partial R_{p}}{\partial \alpha^{1}} \\
\frac{\partial R_{p}}{\partial \alpha^{2}} \\
R_{p n}
\end{array}\right)
$$


where

$$
\begin{aligned}
R_{p}= & \frac{f_{n}}{J}+2\left[\frac{\partial v^{i}}{\partial x^{j}}\right] n^{j} n^{i} \\
R_{p n}= & \frac{1}{J}\left(\frac{\partial \tilde{f}^{1}}{\partial \alpha^{1}}+\frac{\partial \tilde{f}^{2}}{\partial \alpha^{2}}\right)+2 \frac{\partial}{\partial \tau}\left(\left[\frac{\partial v^{i}}{\partial x^{j}}\right]\right) n^{i} \tau^{j} \\
& +2 \frac{\partial}{\partial b}\left(\left[\frac{\partial v^{i}}{\partial x^{j}}\right]\right) n^{i} b^{j}-\frac{[\rho]}{[\mu]} \frac{\partial q^{i}}{\partial t} n^{i} \\
& +\left[\frac{\partial v^{i}}{\partial x^{j}}\right]\left(\frac{\partial}{\partial \tau}\left(n^{j} \tau^{i}+n^{i} \tau^{j}\right)+\frac{\partial}{\partial b}\left(n^{j} b^{i}+n^{i} b^{j}\right)\right)
\end{aligned}
$$

Differentiating Eq. (29) with respect to $\alpha^{n} \quad(n=1,2)$, we obtain

$$
\left[\frac{\partial^{2} v^{i}}{\partial x^{j} \partial x^{k}}\right] \tau_{m}^{j} \tau_{n}^{k}=\frac{\partial^{2} q^{i}}{\partial \alpha^{m} \partial \alpha^{n}}-\left[\frac{\partial v^{i}}{\partial x^{j}}\right] \frac{\partial \tau_{m}^{j}}{\partial \alpha^{n}} .
$$

Differentiating Eq. (30) with respect to $\alpha^{m}(m=1,2)$ leads to

$$
\begin{aligned}
& {\left[\frac{\partial^{2} v^{i}}{\partial x^{j} \partial x^{k}}\right] n^{j} \tau_{m}^{k}+\left[\frac{\partial^{2} v^{j}}{\partial x^{i} \partial x^{k}}\right] n^{j} \tau_{m}^{k}} \\
& =\frac{\partial R_{v}^{i}}{\partial \alpha^{m}}-\left[\frac{\partial v^{i}}{\partial x^{j}}\right] \frac{\partial n^{j}}{\partial \alpha^{m}}-\left[\frac{\partial v^{j}}{\partial x^{i}}\right] \frac{\partial n^{j}}{\partial \alpha^{m}} .
\end{aligned}
$$

From Eq. (27), it follows that

$$
\left[\frac{\partial^{2} v^{i}}{\partial x^{j} \partial x^{j}}\right]=\left[\frac{\partial p}{\partial x^{i}}\right]+\frac{[\rho]}{[\mu]} \frac{\partial q^{i}}{\partial t} .
$$

Combining Eqs. (37)-(39), the following set of equations is obtained.

$$
\begin{aligned}
\left(\begin{array}{lll}
C_{11} & C_{12} & C_{13} \\
C_{21} & C_{22} & C_{23} \\
C_{31} & C_{32} & C_{33}
\end{array}\right)\left(\begin{array}{l}
X_{1} \\
X_{2} \\
X_{3}
\end{array}\right)=\left(\begin{array}{l}
A_{1} \\
A_{2} \\
A_{3}
\end{array}\right) \\
-\left(\begin{array}{lll}
B_{11} & B_{12} & B_{13} \\
B_{21} & B_{22} & B_{23} \\
B_{31} & B_{32} & B_{33}
\end{array}\right)\left(\begin{array}{l}
D_{1} \\
D_{2} \\
D_{3}
\end{array}\right),
\end{aligned}
$$

where the block matrices in the first line of the expression above are given as follows:

$$
X_{i}=\left(\begin{array}{l}
{\left[\frac{\partial^{2} v^{i}}{\partial x^{1} \partial x^{1}}\right]} \\
{\left[\frac{\partial^{2} v^{i}}{\partial x^{1} \partial x^{2}}\right]} \\
{\left[\frac{\partial^{2} v^{i}}{\partial x^{1} \partial x^{3}}\right]} \\
{\left[\frac{\partial^{2} v^{i}}{\partial x^{2} \partial x^{2}}\right]} \\
{\left[\frac{\partial^{2} v^{i}}{\partial x^{2} \partial x^{3}}\right]} \\
{\left[\frac{\partial^{2} v^{i}}{\partial x^{3} \partial x^{3}}\right]}
\end{array}\right), \quad\left(\begin{array}{l}
\left.\frac{\partial v^{i}}{\partial x^{1}}\right] \\
{\left[\frac{\partial v^{i}}{\partial x^{2}}\right]} \\
{\left[\frac{\partial v^{i}}{\partial x^{3}}\right]}
\end{array}\right),
$$

$$
A_{i}=\left(\begin{array}{c}
\frac{\partial^{2} q^{i}}{\partial \alpha^{1} \partial \alpha^{1}} \\
\frac{\partial^{2} q^{i}}{\partial \alpha^{1} \partial \alpha^{2}} \\
\frac{\partial^{2} q^{i}}{\partial \alpha^{2} \partial \alpha^{2}} \\
\frac{\partial R_{v}^{i}}{\partial \alpha^{1}} \\
\frac{\partial R_{v}^{i}}{\partial \alpha^{2}} \\
{\left[\frac{\partial p}{\partial x^{i}}\right]+\frac{[\rho]}{[\mu]} \frac{\partial q^{i}}{\partial t}}
\end{array}\right)
$$

$$
C_{11}=\left(\begin{array}{ccc}
\left(\tau_{1}^{1}\right)^{2} & 2 \tau_{1}^{1} \tau_{1}^{2} & 2 \tau_{1}^{1} \tau_{1}^{3} \\
\tau_{1}^{1} \tau_{2}^{1} & \tau_{1}^{1} \tau_{2}^{2}+\tau_{1}^{2} \tau_{2}^{1} & \tau_{1}^{1} \tau_{2}^{3}+\tau_{1}^{3} \tau_{2}^{1} \\
\left(\tau_{2}^{1}\right)^{2} & 2 \tau_{2}^{1} \tau_{2}^{2} & 2 \tau_{2}^{1} \tau_{2}^{3} \\
2 n^{1} \tau_{1}^{1} & 2 n^{1} \tau_{1}^{2}+n^{2} \tau_{1}^{1} & 2 n^{1} \tau_{1}^{3}+n^{3} \tau_{1}^{1} \\
2 n^{1} \tau_{2}^{1} & 2 n^{1} \tau_{2}^{2}+n^{2} \tau_{2}^{1} & 2 n^{1} \tau_{2}^{3}+n^{3} \tau_{2}^{1} \\
1 & 0 & 0 \\
\left(\tau_{1}^{2}\right)^{2} & 2 \tau_{1}^{2} \tau_{1}^{3} & \left(\tau_{1}^{3}\right)^{2} \\
\tau_{1}^{2} \tau_{2}^{2} & \tau_{1}^{2} \tau_{2}^{3}+\tau_{1}^{3} \tau_{2}^{2} & \tau_{1}^{3} \tau_{2}^{3} \\
\left(\tau_{2}^{2}\right)^{2} & 2 \tau_{2}^{2} \tau_{2}^{3} & \left(\tau_{2}^{3}\right)^{2} \\
n^{2} \tau_{1}^{2} & n^{2} \tau_{1}^{3}+n^{3} \tau_{1}^{2} & n^{3} \tau_{1}^{3} \\
n^{2} \tau_{2}^{2} & n^{2} \tau_{2}^{3}+n^{3} \tau_{2}^{2} & n^{3} \tau_{2}^{3} \\
1 & 0 & 1
\end{array}\right),
$$$$
C_{12}=\left(\begin{array}{cccccc}
0 & 0 & 0 & 0 & 0 & 0 \\
0 & 0 & 0 & 0 & 0 & 0 \\
0 & 0 & 0 & 0 & 0 & 0 \\
n^{2} \tau_{1}^{1} & n^{2} \tau_{1}^{2} & n^{2} \tau_{1}^{3} & 0 & 0 & 0 \\
n^{2} \tau_{2}^{1} & n^{2} \tau_{2}^{2} & n^{2} \tau_{2}^{3} & 0 & 0 & 0 \\
0 & 0 & 0 & 0 & 0 & 0
\end{array}\right),
$$$$
C_{13}=\left(\begin{array}{cccccc}
0 & 0 & 0 & 0 & 0 & 0 \\
0 & 0 & 0 & 0 & 0 & 0 \\
0 & 0 & 0 & 0 & 0 & 0 \\
n^{3} \tau_{1}^{1} & n^{3} \tau_{1}^{2} & n^{3} \tau_{1}^{3} & 0 & 0 & 0 \\
n^{3} \tau_{2}^{1} & n^{3} \tau_{2}^{2} & n^{3} \tau_{2}^{3} & 0 & 0 & 0 \\
0 & 0 & 0 & 0 & 0 & 0
\end{array}\right)
$$$$
B_{11}=\left(\begin{array}{ccc}
\frac{\partial \tau_{1}^{1}}{\partial \alpha^{1}} & \frac{\partial \tau_{1}^{2}}{\partial \alpha^{1}} & \frac{\partial \tau_{1}^{3}}{\partial \alpha^{1}} \\
\frac{\partial \tau_{1}^{1}}{\partial \alpha^{2}} & \frac{\partial \tau_{1}^{2}}{\partial \alpha^{2}} & \frac{\partial \tau_{1}^{3}}{\partial \alpha^{2}} \\
\frac{\partial \tau_{2}^{1}}{\partial \alpha^{2}} & \frac{\partial \tau_{2}^{2}}{\partial \alpha^{2}} & \frac{\partial \tau_{2}^{3}}{\partial \alpha^{2}} \\
2 \frac{\partial n^{1}}{\partial \alpha^{1}} & \frac{\partial n^{2}}{\partial \alpha^{1}} & \frac{\partial n^{3}}{\partial \alpha^{1}} \\
2 \frac{\partial n^{1}}{\partial \alpha^{2}} & \frac{\partial n^{2}}{\partial \alpha^{2}} & \frac{\partial n^{3}}{\partial \alpha^{2}} \\
0 & 0 & 0
\end{array}\right),
$$ 


$$
\begin{aligned}
B_{12} & =\left(\begin{array}{ccc}
0 & 0 & 0 \\
0 & 0 & 0 \\
0 & 0 & 0 \\
\frac{\partial n^{2}}{\partial \alpha^{1}} & 0 & 0 \\
\frac{\partial n^{2}}{\partial \alpha^{2}} & 0 & 0 \\
0 & 0 & 0
\end{array}\right), \\
B_{13} & =\left(\begin{array}{ccc}
0 & 0 & 0 \\
0 & 0 & 0 \\
0 & 0 & 0 \\
\frac{\partial n^{3}}{\partial \alpha^{1}} & 0 & 0 \\
\frac{\partial n^{3}}{\partial \alpha^{2}} & 0 & 0 \\
0 & 0 & 0
\end{array}\right) .
\end{aligned}
$$

The other block matrices can also be derived easily.

Now, differentiating Eq. (33) with respect to $\alpha^{n}(n=1,2)$ yields

$$
\left[\frac{\partial^{2} p}{\partial x^{i} \partial x^{j}}\right] \tau_{m}^{i} \tau_{n}^{j}=\frac{\partial^{2} R_{p}}{\partial \alpha^{m} \partial \alpha^{n}}-\left[\frac{\partial p}{\partial x^{i}}\right] \frac{\partial \tau_{m}^{i}}{\partial \alpha^{n}} .
$$

Differentiating Eq. (24) with respect to $\alpha^{m} \quad(m=1,2)$ yields

$$
\left[\frac{\partial^{2} p}{\partial x^{i} \partial x^{j}}\right] n^{i} \tau_{m}^{j}=\frac{\partial R_{p n}}{\partial \alpha^{m}}-\left[\frac{\partial p}{\partial x^{i}}\right] \frac{\partial n^{i}}{\partial \alpha^{m}} .
$$

From Eq. (28), it follows that

$$
\left[\frac{\partial^{2} p}{\partial x^{i} \partial x^{i}}\right]=-\left[\frac{\rho}{\mu^{2}} \frac{\partial v^{j}}{\partial x^{i}} \frac{\partial v^{i}}{\partial x^{j}}\right] .
$$

Eqs. (48)-(50) form the following set of equations.

$$
\left.\begin{array}{ccc}
\left(\tau_{1}^{1}\right)^{2} & 2 \tau_{1}^{1} \tau_{1}^{2} & 2 \tau_{1}^{1} \tau_{1}^{3} \\
\tau_{1}^{1} \tau_{2}^{1} & \tau_{1}^{1} \tau_{2}^{2}+\tau_{1}^{2} \tau_{2}^{1} & \tau_{1}^{1} \tau_{2}^{3}+\tau_{1}^{3} \tau_{2}^{1} \\
\left(\tau_{2}^{1}\right)^{2} & 2 \tau_{2}^{1} \tau_{2}^{2} & 2 \tau_{2}^{1} \tau_{2}^{3} \\
n^{1} \tau_{1}^{1} & n^{1} \tau_{1}^{2}+n^{2} \tau_{1}^{1} & n^{1} \tau_{1}^{3}+n^{3} \tau_{1}^{1} \\
n^{1} \tau_{2}^{1} & n^{1} \tau_{2}^{2}+n^{2} \tau_{2}^{1} & n^{1} \tau_{2}^{3}+n^{3} \tau_{2}^{1} \\
1 & 0 & 0 \\
\left.\tau_{1}^{2}\right)^{2} & 2 \tau_{1}^{2} \tau_{1}^{3} & \left(\tau_{1}^{3}\right)^{2} \\
\tau_{1}^{2} \tau_{2}^{2} & \tau_{1}^{2} \tau_{2}^{3}+\tau_{1}^{3} \tau_{2}^{2} & \tau_{1}^{3} \tau_{2}^{3} \\
\left(\tau_{2}^{2}\right)^{2} & 2 \tau_{2}^{2} \tau_{2}^{3} & \left(\tau_{2}^{3}\right)^{2} \\
n^{2} \tau_{1}^{2} & n^{2} \tau_{1}^{3}+n^{3} \tau_{1}^{2} & n^{3} \tau_{1}^{3} \\
n^{2} \tau_{2}^{2} & n^{2} \tau_{2}^{3}+n^{3} \tau_{2}^{2} & n^{3} \tau_{2}^{3} \\
1 & 0 & 1
\end{array}\right) \times\left(\begin{array}{l}
{\left[\frac{\partial^{2} p}{\partial x^{1} \partial x^{1}}\right]} \\
{\left[\frac{\partial^{2} p}{\partial x^{1} \partial x^{2}}\right]} \\
{\left[\frac{\partial^{2} p}{\partial x^{1} \partial x^{3}}\right]} \\
{\left[\frac{\partial^{2} p}{\partial x^{2} \partial x^{2}}\right]} \\
{\left[\frac{\partial^{2} p}{\partial x^{2} \partial x^{3}}\right]} \\
{\left[\frac{\partial^{2} p}{\partial x^{3} \partial x^{3}}\right]}
\end{array}\right)
$$

$$
=\left(\begin{array}{c}
\frac{\partial^{2} R_{p}}{\partial \alpha^{1} \partial \alpha^{1}} \\
\frac{\partial^{2} R_{p}}{\partial \alpha^{1} \partial \alpha^{2}} \\
\frac{\partial^{2} R_{p}}{\partial \alpha^{2} \partial \alpha^{2}} \\
\frac{\partial R_{p n}}{\partial \alpha^{1}} \\
\frac{\partial R_{p n}}{\partial \alpha^{2}} \\
R_{p l}
\end{array}\right)-\left(\begin{array}{ccc}
\frac{\partial \tau_{1}^{1}}{\partial \alpha^{1}} & \frac{\partial \tau_{1}^{2}}{\partial \alpha^{1}} & \frac{\partial \tau_{1}^{3}}{\partial \alpha^{1}} \\
\frac{\partial \tau_{1}^{1}}{\partial \alpha^{2}} & \frac{\partial \tau_{1}^{2}}{\partial \alpha^{2}} & \frac{\partial \tau_{1}^{3}}{\partial \alpha^{2}} \\
\frac{\partial \tau_{2}^{1}}{\partial \alpha^{2}} & \frac{\partial \tau_{2}^{2}}{\partial \alpha^{2}} & \frac{\partial \tau_{2}^{3}}{\partial \alpha^{2}} \\
\frac{\partial n^{1}}{\partial \alpha^{1}} & \frac{\partial n^{2}}{\partial \alpha^{1}} & \frac{\partial n^{3}}{\partial \alpha^{1}} \\
\frac{\partial n^{1}}{\partial \alpha^{2}} & \frac{\partial n^{2}}{\partial \alpha^{2}} & \frac{\partial n^{3}}{\partial \alpha^{2}} \\
0 & 0 & 0
\end{array}\right) \times\left(\begin{array}{c}
{\left[\frac{\partial p}{\partial x^{1}}\right]} \\
{\left[\frac{\partial p}{\partial x^{2}}\right]} \\
{\left[\frac{\partial p}{\partial x^{3}}\right]}
\end{array}\right),
$$

where $R_{p l}=-\left[\frac{\rho}{\mu^{2}} \frac{\partial v^{j}}{\partial x^{i}} \frac{\partial v^{i}}{\partial x^{j}}\right]$.

Given $q^{i}$, Eqs. (31), (34), (40), and (51) can be solved for the other jump conditions. In addition to those equations and Eq. (26), we also have the following jump condition

$$
\left[\frac{v^{i}}{\mu}\right]=0
$$

which is a result of the continuity of the velocity. Given $q^{i}$, and the jump conditions for the first order derivatives, $\left(v^{i} / \mu\right)^{ \pm}$can be obtained using an interpolation scheme. Thus, the equations can be solved numerically for all of the unknowns by an iterative method described in subsection 6.1 .

\section{Numerical Application \\ 6.1 Solving for the jump conditions and dis- cretization of the governing equations}

In this section, the scaled velocity, denoted by $\boldsymbol{v}=$ $(u, v, w)$, and the pressure are discretized on a fixed Cartesian regular grid as shown schematically in Fig. 4. The singular surface $S$ is represented by Lagrangian markers. Coordinates and other quantities at points of the surface other than the Lagrangian markers are obtained using a suitable interpolation scheme, such as the cubic spline interpolation. In general, the grid lines intersect the singular surface $S$ at points that do not coincide with the grid points. These points, such as $K$ and $L$ in Fig. 4 , are called interfacial points. Grid points adjacent to an interfacial point, such as $M$ and $N$, are called irregular grid points. As described in sections 1 and 2, obtaining the jump conditions at interfacial points and modifying the finite differences at irregular grid points constitute the essential parts in constructing an IIM method for simulating multiphase flows.

Consider point $K$ in Fig. 4. It can be shown that the values of the scaled velocity at $K$ are given by the following interpolation formulae [8]

$$
\begin{aligned}
\boldsymbol{v}_{K}^{-}= & \frac{h^{-} \boldsymbol{v}_{N}+h^{+} \boldsymbol{v}_{M}}{h}-\frac{h^{-}}{h} \boldsymbol{q}_{K} \\
& -\frac{h^{-} h^{+}}{h}\left[\frac{\partial \boldsymbol{v}}{\partial x}\right]_{K}+O\left(h^{2}\right),
\end{aligned}
$$




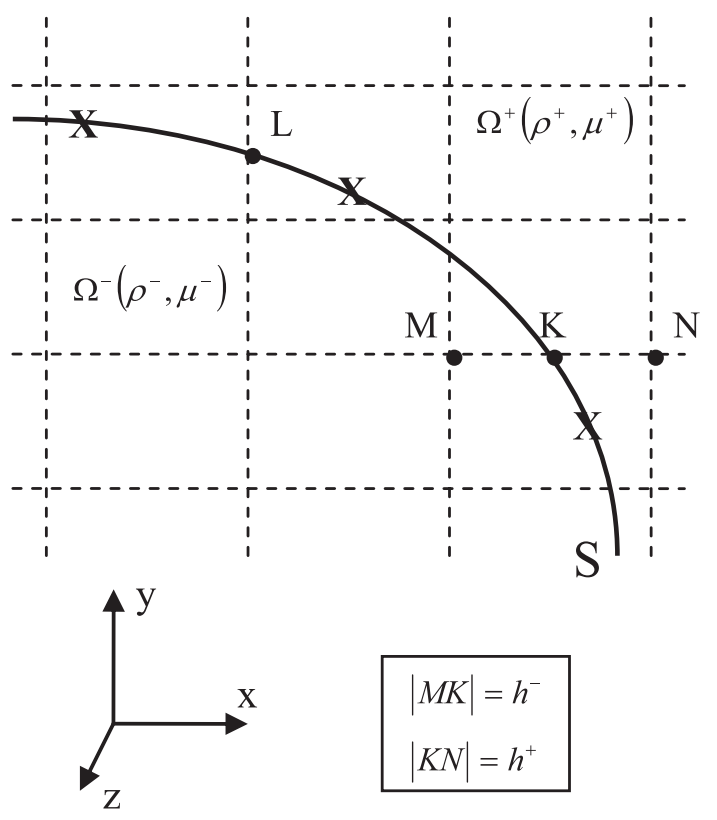

Fig. 4 Schematic view of a part of the Cartesian regular grid near the singular surface. Dashed lines represent grid lines. $X$ 's represent Lagrangian markers.

$$
\begin{aligned}
\boldsymbol{v}_{K}^{+}= & \frac{h^{-} \boldsymbol{v}_{N}+h^{+} \boldsymbol{v}_{M}}{h}+\frac{h^{+}}{h} \boldsymbol{q}_{K} \\
& -\frac{h^{-} h^{+}}{h}\left[\frac{\partial \boldsymbol{v}}{\partial x}\right]_{K}+O\left(h^{2}\right),
\end{aligned}
$$

where $\boldsymbol{q}=\left(q_{x}, q_{y}, q_{z}\right)=([u],[v],[w])$ denotes the augmented variables, and $h=h^{+}+h^{-}$. Now, substituting Eqs. (53) and (54) into Eq. (52), treating $\boldsymbol{q}_{K}$ as the unknown, the following equation is obtained.

$$
\begin{aligned}
\boldsymbol{q}_{K}= & \frac{[\mu]}{h^{+} \mu^{-}+h^{-} \mu^{+}}\left(h^{-} \boldsymbol{v}_{N}+h^{+} \boldsymbol{v}_{M}\right) \\
& -\frac{[\mu] h^{-} h^{+}}{h^{+} \mu^{-}+h^{-} \mu^{+}}\left[\frac{\partial \boldsymbol{v}}{\partial x}\right]_{K}+O\left(h^{2}\right) .
\end{aligned}
$$

Hence, to obtain the jumps of the first derivatives of the scaled velocity and the augmented variables, Eq. (31) and equations similar to Eq. (55) (which will be called collectively as Eq. (55)) must be solved simultaneously at all interfacial points. This can be done by an iterative method. Initially, guessed values of $\boldsymbol{q}$ are substituted into the right hand side (RHS) of Eq. (31). Eq. (31) is then

solved for $[\partial v / \partial x],[\partial v / \partial y]$, and $[\partial v / \partial z]$. Then, substituting the obtained values into the RHS of Eq. (55), new guessed values of $\boldsymbol{q}$ are obtained. The entire procedure is iterated until the values of $\boldsymbol{q}$ converge. After obtaining the augmented variables and the jumps of the first derivatives of the scaled velocity, Eqs. (34), (40), and (51) can be solved for the remaining jump conditions. Thus, the systems of equations in the previous section can be solved numerically for all of the unknowns.

Next, an outline of the discretization of the governing equations is described. Spatial derivatives in the Navier
Stokes equation Eq. (27) and the Poisson equation for pressure with terms containing $\nabla \cdot \boldsymbol{v}$ (given by Eq. (77) in Appendix $\mathrm{C}$ for the $2 \mathrm{D}$ case) are approximated by finite differences. Using the jump conditions obtained by the procedure described in the previous paragraph, finite differences based on Eqs. (6) and (7) are applied at irregular grid points. At regular grid points, central finite differences are applied. The scaled velocity and the pressure can be advanced in time by well-known methods such as a combination of the MAC scheme and the Runge-Kutta scheme. Solving Eq. (4), the coordinates of the Lagrangian markers are also advanced in time simultaneously with the scaled velocity and the pressure.

Note that it is assumed that the singular force density is known a priori or can be calculated from the local configuration of the singular surface. We are particularly interested in problems with singular force densities of the latter kind for our future work. The normal force density (determined by the curvature) resulting from surface tension and the force density resulting from elastic tensions (determined by the strains) are typical examples of such force densities. Quantities such as local curvature and local strains can be calculated numerically because the singular surface is represented by Lagrangian markers and a suitable interpolation scheme. The values can then be used to obtain the singular force densities. Thus, it is possible to model singular force densities such as those acting on fluid-fluid interfaces or elastic membranes in the current representation of the singular surface.

\subsection{Numerical test}

A numerical test is performed to verify that (1) the procedure for obtaining the jump conditions, and (2) the finite difference approximations Eqs. (6) and (7) yield correct results. We consider the shear flow described schematically in Fig. 5. Coordinate axes are taken as shown in the figure. Two fluids, fluids 1 and 2, with different viscosities and mass densities are separated by an infinite rigid plane (plane B) parallel to the $x-z$ plane and bounded by infinite rigid planes $\mathrm{A}$ and $\mathrm{C}$ parallel to $\mathrm{B}$. Plane B moves with (ordinary) velocity $U$ in the $x$ direction, while planes $\mathrm{A}$ and $\mathrm{C}$ are stationary. The steady flow solution of this problem is well known as the Couette flow. In the Couette flow, the scaled velocity $u(y)$ of each fluid varies linearly as shown in Fig. 6a, and the pressure is constant everywhere. From this analytical solution, the tangential singular force density on plane B can be obtained.

The region between planes $\mathrm{A}$ and $\mathrm{C}$ is discretized in the $y$ direction. At the grid points $y_{i}$, the analytical solution $u\left(y_{i}\right)$ is obtained. Substituting the tangential force density into the equations for the jump conditions in two dimensions given in Appendix C, jump conditions that agree with the analytical results are obtained. The augmented variable is then computed using Eq. (55). Finally, using the obtained values of the jump conditions and the augmented 
C

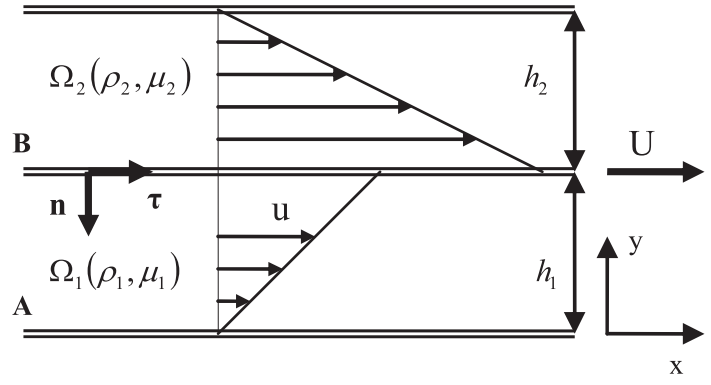

Fig. 5 Shear flow between parallel infinite rigid planes. Plane A is located at $y=0$. Planes $\mathrm{A}$ and $\mathrm{C}$ are stationary. Plane B moves with velocity $U$ in the $\mathrm{x}$ direction.

(a)
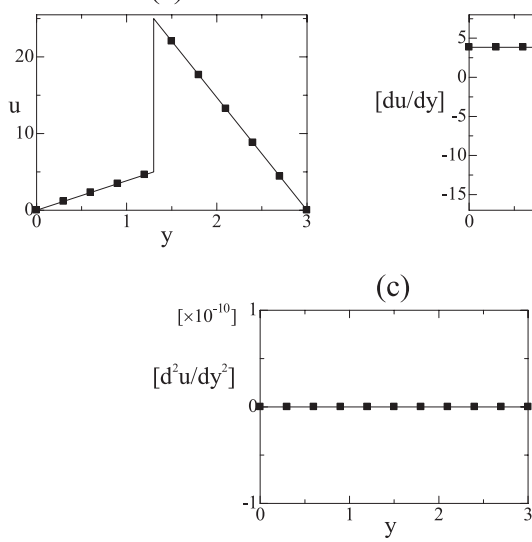

(c) (b)

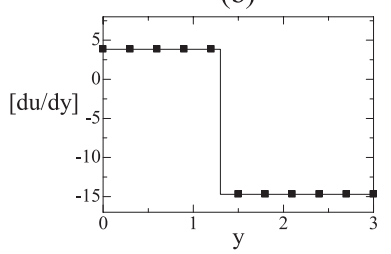

Fig. 6 Comparisons between analytical and numerical results for the flow described in Fig. 5. Panels (a), (b), and (c) show profiles of the scaled velocity, its first derivative, and its second derivative, respectively. Horizontal axes show the y coordinate. Solid lines and dots represent analytical and numerical results, respectively.

variable, the finite differences given by Eqs. (6) and (7) are used to obtain the first and second derivatives of the scaled velocity at the irregular grid points. The numerical results for parameter values $\mu_{1}=1, \mu_{2}=5, h_{1}=1.3, h_{2}=1.7$, $U=5$, and grid interval $\Delta y=0.3$ are shown in Fig. 6. Figures $6 \mathrm{a}, \mathrm{b}$, and $\mathrm{c}$ show the profiles of the scaled velocity, its first derivative, and its second derivative, respectively. It can be seen that the numerical derivatives agree well with the analytical derivatives at all grid points including the irregular points. These numerical results show that our algorithm is suitable for computations of two fluids with different densities and viscosities separated by a wall.

\section{Concluding Remarks}

Jump conditions in multiphase incompressible flows with singular forces and discontinuities in viscosity and density have been derived. The results are sets of equations that are solvable numerically by an iterative method. These jump conditions can be used to develop an IIM scheme for simulating multiphase flows with discontinuous viscosity and density. A numerical code implementing the discretization schemes described in subsection 6.1 is currently being developed.

Ito and $\mathrm{Li}$ [10] derived the jump conditions in Stokes flow with singular forces and discontinuous viscosity. Other than in the inclusion of density jump, our derivation is more general and conclusive than their, in the sense that our results are explicit relations in a three-dimensional fixed Cartesian coordinate system, whereas their results were explicit relations in a two-dimensional special coordinate system.

We finally remark that, although the current formulation is for incompressible flows, jump conditions for compressible flows, thermal quantities, and/or magnetic field can also be derived in a manner similar to our derivation reported in this article. Extension of the IIM to thermal plasma simulations is also under consideration.

\section{Appendix A. Derivation of Eqs. (21) and (22)}

The left hand side of Eq. (20) vanishes since the integrand is bounded. By the definition of $F^{i}$, the first term on the right hand side (RHS) of Eq. (20) becomes

$$
\lim _{\varepsilon \rightarrow 0} \int_{\delta V} F^{i} d V=f^{i} \delta \alpha^{1} \delta \alpha^{2}
$$

Applying the Generalized Gauss Theorem [7] to the second term on the RHS of Eq. (20) yields

$$
\begin{gathered}
\lim _{\varepsilon \rightarrow 0} \int_{\delta V} \frac{\partial}{\partial x^{j}}\left\{-p \delta^{i j}+\mu\left(\frac{\partial u^{i}}{\partial x^{j}}+\frac{\partial u^{j}}{\partial x^{i}}\right)\right\} \mathrm{d} V \\
=\left(-[p] n^{i}+\left[\mu \frac{\partial u^{i}}{\partial x^{j}}\right] n^{j}+\left[\mu \frac{\partial u^{j}}{\partial x^{i}}\right] n^{j}\right) \delta S .
\end{gathered}
$$

Substituting the above results into Eq. (20), the following equation is obtained.

$$
-[p] n^{i}+\left[\mu \frac{\partial u^{i}}{\partial x^{j}}\right] n^{j}+\left[\mu \frac{\partial u^{j}}{\partial x^{i}}\right] n^{j}+\frac{f^{i}}{J}=0
$$

Multiplying this equation by $n^{i}$ leads to Eq. (21). Eq. (22) is obtained by substituting Eq. (21) back into Eq. (A.3).

\section{Appendix B. Derivation of eq. (24)}

The term on the left hand side (LHS) of Eq. (23) can be written as

$$
\begin{gathered}
\lim _{\varepsilon \rightarrow 0} \int_{V s} \phi \frac{\partial^{2} p}{\partial x^{i} \partial x^{i}} \mathrm{~d} V=\lim _{\varepsilon \rightarrow 0} \int_{V s} \frac{\partial}{\partial x^{i}}\left(\phi \frac{\partial p}{\partial x^{i}}\right) \mathrm{d} V \\
-\lim _{\varepsilon \rightarrow 0} \int_{V s} \frac{\partial}{\partial x^{i}}\left(\frac{\partial \phi}{\partial x^{i}} p\right) \mathrm{d} V+\lim _{\varepsilon \rightarrow 0} \int_{V s} \frac{\partial^{2} \phi}{\partial x^{i} \partial x^{i}} p \mathrm{~d} V .
\end{gathered}
$$

The third term on the right hand side (RHS) of the above equation vanishes because the integrand is bounded. Applying the Generalized Gauss Theorem (GGT) to the first 
and second terms on the RHS, we obtain

$$
\begin{aligned}
\lim _{\varepsilon \rightarrow 0} \int_{V_{s}} \phi \frac{\partial^{2} p}{\partial x^{i} \partial x^{i}} \mathrm{~d} V= & \int_{S} \phi\left[\frac{\partial p}{\partial x^{i}}\right] n^{i} \mathrm{~d} S \\
& -\int_{S}[p] \frac{\partial \phi}{\partial n} \mathrm{~d} S .
\end{aligned}
$$

By the definition of $F^{i}$, the first term on the RHS of Eq. (23) becomes

$$
\begin{aligned}
\lim _{\varepsilon \rightarrow 0} \int_{V_{s}} \phi \frac{\partial F^{i}}{\partial x^{i}} \mathrm{~d} V & =-\lim _{\varepsilon \rightarrow 0} \int_{V_{s}} \frac{\partial \phi}{\partial x^{i}} F^{i} \mathrm{~d} V \\
& =-\int_{S} f^{i} \frac{\partial \phi}{\partial x^{i}} \mathrm{~d} \alpha^{1} \mathrm{~d} \alpha^{2} .
\end{aligned}
$$

Applying the coordinate transformation, this can be written as

$$
\begin{aligned}
-\int_{S} f^{i} \frac{\partial \phi}{\partial x^{i}} \mathrm{~d} \alpha^{1} \mathrm{~d} \alpha^{2}= & -\int_{S} f^{k} n^{k} \frac{\partial \phi}{\partial n} \mathrm{~d} \alpha^{1} \mathrm{~d} \alpha^{2} \\
& -\int_{S}\left(\tilde{f}^{1} \frac{\partial \phi}{\partial \alpha^{1}}+\tilde{f}^{2} \frac{\partial \phi}{\partial \alpha^{2}}\right) \mathrm{d} \alpha^{1} \mathrm{~d} \alpha^{2} \\
= & -\int_{S} f^{k} n^{k} \frac{\partial \phi}{\partial n} \mathrm{~d} \alpha^{1} \mathrm{~d} \alpha^{2} \\
& -\int_{S}\left(\frac{\partial}{\partial \alpha^{1}}\left(\tilde{f}^{1} \phi\right)+\frac{\partial}{\partial \alpha^{2}}\left(\tilde{f}^{2} \phi\right)\right) \mathrm{d} \alpha^{1} \mathrm{~d} \alpha^{2} \\
& +\int_{S} \phi\left(\frac{\partial \tilde{f}^{1}}{\partial \alpha^{1}}+\frac{\partial \tilde{f}^{2}}{\partial \alpha^{2}}\right) \mathrm{d} \alpha^{1} \mathrm{~d} \alpha^{2} . \text { (B.4) }
\end{aligned}
$$

Due to the assumption that $S$ is a closed surface, the second term on the last RHS of Eq. (B.4) vanishes. Thus, the following equation is obtained.

$$
\begin{aligned}
\lim _{\varepsilon \rightarrow 0} \int_{V_{s}} \phi \frac{\partial F^{i}}{\partial x^{i}} & \mathrm{~d} V=-\int_{S} f^{k} n^{k} \frac{\partial \phi}{\partial n} \mathrm{~d} \alpha^{1} \mathrm{~d} \alpha^{2} \\
& +\int_{S} \phi\left(\frac{\partial \tilde{f}^{1}}{\partial \alpha^{1}}+\frac{\partial \tilde{f}^{2}}{\partial \alpha^{2}}\right) \mathrm{d} \alpha^{1} \mathrm{~d} \alpha^{2} .
\end{aligned}
$$

Next, we calculate the second term on the RHS of Eq. (23).

$$
\begin{aligned}
\lim _{\varepsilon \rightarrow 0} & \int_{V_{s}} \phi \frac{\partial}{\partial x^{i}}\left\{\frac{\partial}{\partial x^{j}}\left(\mu \frac{\partial u^{i}}{\partial x^{j}}\right)+\frac{\partial}{\partial x^{j}}\left(\mu \frac{\partial u^{j}}{\partial x^{i}}\right)\right\} \mathrm{d} V \\
= & \lim _{\varepsilon \rightarrow 0} \int_{V_{s}} \phi \frac{\partial}{\partial x^{i}}\left\{\frac{\partial}{\partial x^{j}}\left(\mu \frac{\partial u^{i}}{\partial x^{j}}\right)\right\} \mathrm{d} V \\
& +\lim _{\varepsilon \rightarrow 0} \int_{V_{s}} \phi \frac{\partial}{\partial x^{i}}\left\{\frac{\partial}{\partial x^{j}}\left(\mu \frac{\partial u^{j}}{\partial x^{i}}\right)\right\} \mathrm{d} V .
\end{aligned}
$$

The first term on the RHS of this equation can be written as

$$
\begin{aligned}
\lim _{\varepsilon \rightarrow 0} & \int_{V_{s}} \phi \frac{\partial}{\partial x^{i}}\left\{\frac{\partial}{\partial x^{j}}\left(\mu \frac{\partial u^{i}}{\partial x^{j}}\right)\right\} \mathrm{d} V \\
= & \lim _{\varepsilon \rightarrow 0} \int_{V_{s}} \frac{\partial}{\partial x^{i}}\left\{\phi \frac{\partial}{\partial x^{j}}\left(\mu \frac{\partial u^{i}}{\partial x^{j}}\right)\right\} \mathrm{d} V \\
& -\lim _{\varepsilon \rightarrow 0} \int_{V_{s}} \frac{\partial}{\partial x^{j}}\left\{\frac{\partial \phi}{\partial x^{i}} \mu \frac{\partial u^{i}}{\partial x^{j}}\right\} \mathrm{d} V \\
& +\lim _{\varepsilon \rightarrow 0} \int_{V_{s}} \frac{\partial^{2} \phi}{\partial x^{i} \partial x^{j}} \mu \frac{\partial u^{i}}{\partial x^{j}} \mathrm{~d} V .
\end{aligned}
$$

The last term on the RHS of Eq. (B.7) vanishes since the integrand is bounded. Hence, applying the GGT, we obtain

$$
\begin{aligned}
& \lim _{\varepsilon \rightarrow 0} \int_{V_{s}} \phi \frac{\partial}{\partial x^{i}}\left\{\frac{\partial}{\partial x^{j}}\left(\mu \frac{\partial u^{i}}{\partial x^{j}}\right)\right\} \mathrm{d} V \\
& \quad=\int_{S} \phi\left[\mu \frac{\partial^{2} u^{i}}{\partial x^{j} \partial x^{j}}\right] n^{i} \mathrm{~d} S-\int_{S}\left[\mu \frac{\partial u^{i}}{\partial x^{j}}\right] n^{j} \frac{\partial \phi}{\partial x^{i}} \mathrm{~d} S .
\end{aligned}
$$

The second term on the RHS of Eq. (B.6) can be written as

$$
\begin{aligned}
\lim _{\varepsilon \rightarrow 0} & \int_{V_{s}} \phi \frac{\partial}{\partial x^{i}}\left\{\frac{\partial}{\partial x^{j}}\left(\mu \frac{\partial u^{j}}{\partial x^{i}}\right)\right\} \mathrm{d} V \\
= & \int_{S} \phi\left[\mu \frac{\partial^{2} u^{j}}{\partial x^{i} \partial x^{j}}\right] n^{i} \mathrm{~d} S-\int_{S}\left[\mu \frac{\partial u^{j}}{\partial x^{i}}\right] n^{j} \frac{\partial \phi}{\partial x^{i}} \mathrm{~d} S \\
& \quad+\lim _{\varepsilon \rightarrow 0} \int_{V s} \frac{\partial^{2} \phi}{\partial x^{i} \partial x^{j}} \mu \frac{\partial u^{j}}{\partial x^{i}} \mathrm{~d} V,
\end{aligned}
$$

where the GGT has been applied to obtain the first and second terms on the RHS. The first term on the RHS of this equation equals zero due to the incompressibility condition, and the last term vanishes because the integrand is bounded. Therefore, we obtain

$$
\begin{gathered}
\lim _{\varepsilon \rightarrow 0} \int_{V_{s}} \phi \frac{\partial}{\partial x^{i}}\left\{\frac{\partial}{\partial x^{j}}\left(\mu \frac{\partial u^{j}}{\partial x^{i}}\right)\right\} \mathrm{d} V \\
=-\int_{S}\left[\mu \frac{\partial u^{j}}{\partial x^{i}}\right] n^{j} \frac{\partial \phi}{\partial x^{i}} \mathrm{~d} S
\end{gathered}
$$

Using Eq. (11), the RHS of Eq. (B.8) and Eq. (B.10) can be calculated further. First, it is easy to see that

$$
\begin{aligned}
& \int_{S}\left[\mu \frac{\partial u^{i}}{\partial x^{j}}\right] n^{j} \frac{\partial \phi}{\partial x^{i}} \mathrm{~d} S \\
&=\int_{S}\left[\mu \frac{\partial u^{i}}{\partial x^{j}}\right] n^{j} n^{i} \frac{\partial \phi}{\partial n} \mathrm{~d} S \\
&+\int_{S}\left[\mu \frac{\partial u^{i}}{\partial x^{j}}\right] n^{j} \tau^{i} \frac{\partial \phi}{\partial \tau} \mathrm{d} S+\int_{S}\left[\mu \frac{\partial u^{i}}{\partial x^{j}}\right] n^{j} b^{i} \frac{\partial \phi}{\partial b} \mathrm{~d} S \\
&=\int_{S}\left[\mu \frac{\partial u^{i}}{\partial x^{j}}\right] n^{j} n^{i} \frac{\partial \phi}{\partial n} \mathrm{~d} S \\
&-\int_{S} \phi \frac{\partial}{\partial \tau}\left\{\left[\mu \frac{\partial u^{i}}{\partial x^{j}}\right] n^{j} \tau^{i}\right\} \mathrm{d} S \\
&-\int_{S} \phi \frac{\partial}{\partial b}\left\{\left[\mu \frac{\partial u^{i}}{\partial x^{j}}\right] n^{j} b^{i}\right\} \mathrm{d} S \\
&+\int_{S} \frac{\partial}{\partial \tau}\left\{\left[\mu \frac{\partial u^{i}}{\partial x^{j}}\right] n^{j} \tau^{i} \phi\right\} \mathrm{d} S \\
&+\int_{S} \frac{\partial}{\partial b}\left\{\left[\mu \frac{\partial u^{i}}{\partial x^{j}}\right] n^{j} b^{i} \phi\right\} \mathrm{d} S .
\end{aligned}
$$

The sum of the last two terms of this equation vanishes because $S$ is a closed surface. It follows that

$$
\begin{gathered}
\int_{S}\left[\mu \frac{\partial u^{i}}{\partial x^{j}}\right] n^{j} \frac{\partial \phi}{\partial x^{i}} \mathrm{~d} S=\int_{S}\left[\mu \frac{\partial u^{i}}{\partial x^{j}}\right] n^{j} n^{i} \frac{\partial \phi}{\partial n} \mathrm{~d} S \\
-\int_{S} \phi\left\{\left[\mu \frac{\partial^{2} u^{i}}{\partial x^{j} \partial x^{k}}\right] n^{j}\left(\tau^{i} \tau^{k}+b^{i} b^{k}\right)\right\} \mathrm{d} S \\
-\int_{S} \phi\left\{\left[\mu \frac{\partial u^{i}}{\partial x^{j}}\right]\left(\frac{\partial}{\partial \tau}\left(n^{j} \tau^{i}\right)+\frac{\partial}{\partial b}\left(n^{j} b^{i}\right)\right)\right\} \mathrm{d} S .
\end{gathered}
$$


Using Eq. (11) again, and taking the incompressibility condition into account, this equation becomes

$$
\begin{aligned}
& \int_{S}\left[\mu \frac{\partial u^{i}}{\partial x^{j}}\right] n^{j} \frac{\partial \phi}{\partial x^{i}} \mathrm{~d} S=\int_{S}\left[\mu \frac{\partial u^{i}}{\partial x^{j}}\right] n^{j} n^{i} \frac{\partial \phi}{\partial n} \mathrm{~d} S \\
& +\int_{S} \phi\left[\mu \frac{\partial^{2} u^{i}}{\partial x^{j} \partial x^{k}}\right] n^{j} n^{i} n^{k} \mathrm{~d} S \\
& -\int_{S} \phi\left\{\left[\mu \frac{\partial u^{i}}{\partial x^{j}}\right]\left(\frac{\partial}{\partial \tau}\left(n^{j} \tau^{i}\right)+\frac{\partial}{\partial b}\left(n^{j} b^{i}\right)\right)\right\} \mathrm{d} S .
\end{aligned}
$$

By following arguments similar to those in the previous paragraph, it can be shown that the RHS of Eq. (B.10) becomes

$$
\begin{aligned}
& \int_{S}\left[\mu \frac{\partial u^{j}}{\partial x^{i}}\right] n^{j} \frac{\partial \phi}{\partial x^{i}} \mathrm{~d} S=\int_{S}\left[\mu \frac{\partial u^{j}}{\partial x^{i}}\right] n^{j} n^{i} \frac{\partial \phi}{\partial n} \mathrm{~d} S \\
& -\int_{S} \phi\left[\mu \frac{\partial^{2} u^{j}}{\partial x^{i} \partial x^{i}}\right] n^{j} \mathrm{~d} S \\
& +\int_{S} \phi\left[\mu \frac{\partial^{2} u^{j}}{\partial x^{i} \partial x^{k}}\right] n^{j} n^{i} n^{k} \mathrm{~d} S \\
& -\int_{S} \phi\left\{\left[\mu \frac{\partial u^{j}}{\partial x^{i}}\right]\left(\frac{\partial}{\partial \tau}\left(n^{j} \tau^{i}\right)+\frac{\partial}{\partial b}\left(n^{j} b^{i}\right)\right)\right\} \mathrm{d} S .
\end{aligned}
$$

Next, we calculate the last term on the RHS of Eq. (23). Using the GGT and Eq. (19), we obtain

$$
\begin{gathered}
\lim _{\varepsilon \rightarrow 0} \int_{V_{s}} \phi \frac{\partial}{\partial x^{i}}\left(\rho \frac{\mathrm{D} u^{i}}{\mathrm{D} t}\right) \mathrm{d} V=-\lim _{\varepsilon \rightarrow 0} \int_{V s} \frac{\partial \phi}{\partial x^{i}} \rho \frac{\mathrm{D} u^{i}}{\mathrm{D} t} \mathrm{~d} V \\
\quad+\lim _{\varepsilon \rightarrow 0} \int_{V s} \frac{\partial}{\partial x^{i}}\left(\phi \rho \frac{\mathrm{D} u^{i}}{\mathrm{D} t}\right) \mathrm{d} V \\
=\int_{S} \phi[\rho] \frac{\mathrm{D} u^{i}}{\mathrm{D} t} n^{i} \mathrm{~d} S,
\end{gathered}
$$

where the first term on the first RHS equals zero since the integrand is bounded.

Substituting Eqs.(B.2), (B.5), (B.6), (B.8), (B.10), (B.13), (B.14), and (B.15) into Eq. (23), and rearranging terms, lead to

$$
\begin{gathered}
\int_{S} \phi \\
\left.-2\left[\frac{\partial p}{\partial x^{i}}\right] n^{i}-\frac{1}{J}\left(\frac{\partial \tilde{f}^{1}}{\partial \alpha^{1}}+\frac{\partial \tilde{f}^{2}}{\partial \alpha^{2}}\right)+[\rho] \frac{\partial^{2} u^{i}}{\partial x^{j} \partial x^{j}}\right] n^{i}+2\left[\mu \frac{\partial^{2} u^{i}}{\partial x^{j} \partial x^{k}}\right] n^{i} n^{i} n^{k} \\
-\left(\left[\mu \frac{\partial u^{i}}{\partial x^{j}}\right]+\left[\mu \frac{\partial u^{j}}{\partial x^{i}}\right]\right)\left(\frac{\partial}{\partial \tau}\left(n^{j} \tau^{i}\right)\right. \\
\left.\left.+\frac{\partial}{\partial b}\left(n^{j} b^{i}\right)\right)\right\} \mathrm{d} S \\
=\int_{S} \frac{\partial \phi}{\partial n}\left\{[p]-\frac{f^{i} n^{i}}{J}-2\left[\mu \frac{\partial u^{i}}{\partial x^{j}}\right] n^{j} n^{i}\right\} \mathrm{d} S . \quad \text { (B. }
\end{gathered}
$$

According to Eq. (21), the RHS of Eq. (B.16) vanishes. Since $\phi$ is arbitrary, it follows that the integrand of the LHS must equal zero. Therefore, the following equation is obtained.

$$
\begin{aligned}
{\left[\frac{\partial p}{\partial x^{i}}\right] n^{i}=} & \frac{1}{J}\left(\frac{\partial \tilde{f}^{1}}{\partial \alpha^{1}}+\frac{\partial \tilde{f}^{2}}{\partial \alpha^{2}}\right)+2\left[\mu \frac{\partial^{2} u^{i}}{\partial x^{j} \partial x^{j}}\right] n^{i} \\
& -2\left[\mu \frac{\partial^{2} u^{i}}{\partial x^{j} \partial x^{k}}\right] n^{i} n^{j} n^{k}
\end{aligned}
$$

$$
\begin{aligned}
& +\left[\mu \frac{\partial u^{i}}{\partial x^{j}}\right]\left(\frac{\partial}{\partial \tau}\left(n^{j} \tau^{i}+n^{i} \tau^{j}\right)\right. \\
& \left.+\frac{\partial}{\partial b}\left(n^{j} b^{i}+n^{i} b^{j}\right)\right)-[\rho] \frac{\mathrm{D} u^{i}}{\mathrm{D} t} n^{i}
\end{aligned}
$$

Applying Eq. (11), this equation becomes Eq. (24).

\section{Appendix C. Jump Conditions in 2D Flows}

In this appendix, we are concerned with twodimensional multiphase flows. A schematic view of the system is given in Fig. 7. It is to be understood that all quantities that appear henceforth are non-dimensionalized. The Cartesian coordinates, the scaled velocity, and the pressure are denoted by $\boldsymbol{x}=(x, y), \boldsymbol{v}=(u, v)$, and $p$, respectively. The coordinates of a point of the singular curve $C$ are denoted by $\boldsymbol{X}\left(\alpha^{1}, t\right)$, where $\alpha^{1}$ is the Lagrangian parameter of the points of the curve at a reference time. The singular force is now defined by $\boldsymbol{F}=\int_{C} \boldsymbol{f}\left(\alpha^{1}, t\right) \delta\left(\boldsymbol{x}-\boldsymbol{X}\left(\alpha^{1}, t\right)\right) \mathrm{d} \alpha^{1}$, where $\boldsymbol{f}\left(\alpha^{1}, t\right)$ is the curve force density, and $\delta\left(\boldsymbol{x}-\boldsymbol{X}\left(\alpha^{1}, t\right)\right)$ is a twodimensional delta function. Geometrical quantities, $\tau_{1}=$ $\partial \boldsymbol{X} / \partial \alpha^{1}, \quad J=\left|\tau_{1}\right|, \quad \tau=\tau_{1} / J$, and $\boldsymbol{n}=\left(\tau_{y},-\tau_{x}\right)$, are also introduced. For $\boldsymbol{x} \notin C$, the Navier-Stokes equation and the Poisson equation for pressure are given by

$$
\begin{aligned}
\frac{\rho}{\mu} \frac{\partial v}{\partial t}+ & \frac{\rho}{\mu^{2}} \nabla \cdot(\boldsymbol{v v})=-\nabla p+\frac{1}{R e} \Delta v, \\
\Delta p=- & \frac{\rho}{\mu} \frac{\partial D}{\partial t}-\frac{\rho}{\mu^{2}} \nabla \cdot(\boldsymbol{v} D) \\
& +2 \frac{\rho}{\mu^{2}}\left(\frac{\partial u}{\partial x} \frac{\partial v}{\partial y}-\frac{\partial u}{\partial y} \frac{\partial v}{\partial x}\right)+\frac{1}{R e} \Delta D,
\end{aligned}
$$

where $R e$ is the Reynolds number, and $D=\nabla \cdot v$.

The systems of equations for the jump conditions in the $2 \mathrm{D}$ case, which can be obtained from our $3 \mathrm{D}$ results by taking one direction as uniform, are:

$$
[u]=q_{x}, \quad[v]=q_{y},
$$

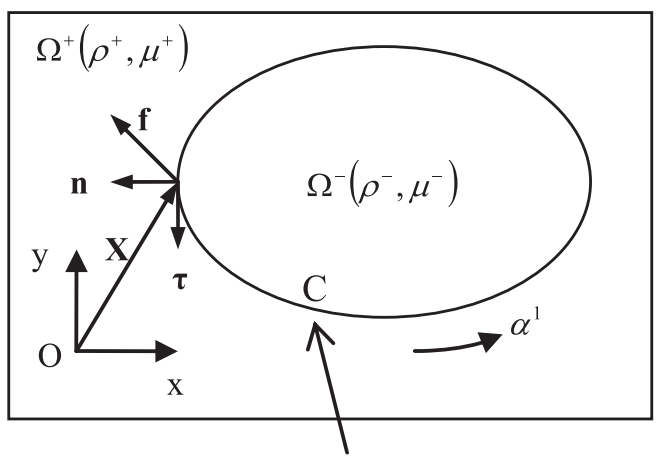

singular curve

Fig. 7 Schematic view of a two-dimensional multiphase flow with singular force. 
$$
\left(\begin{array}{cccc}
\tau_{1 x} & \tau_{1 y} & 0 & 0 \\
2 n_{x} & n_{y} & n_{y} & 0 \\
0 & 0 & \tau_{1 x} & \tau_{1 y} \\
0 & n_{x} & n_{x} & 2 n_{y}
\end{array}\right)\left(\left[\begin{array}{c}
\partial u / \partial x \\
\partial u / \partial y \\
{[\partial v / \partial x} \\
\partial v / \partial y
\end{array}\right]\right)=\left(\begin{array}{c}
\partial q_{x} / \partial \alpha^{1} \\
R_{v x} \\
\partial q_{y} / \partial \alpha^{1} \\
R_{v y}
\end{array}\right),
$$

$[p]=R_{p}$,

$\left(\begin{array}{cc}\tau_{1 x} & \tau_{1 y} \\ n_{x} & n_{y}\end{array}\right)\left(\left[\begin{array}{l}\partial p / \partial x \\ \partial p / \partial y\end{array}\right]\right)=\left(\begin{array}{c}\partial R_{p} / \partial \alpha^{1} \\ R_{p n}\end{array}\right)$

$\left(\begin{array}{ccc}\tau_{1 x}^{2} & 2 \tau_{1 x} \tau_{1 y} & \tau_{1 y}^{2} \\ 2 n_{x} \tau_{1 x} & 2 n_{x} \tau_{1 y}+n_{y} \tau_{1 x} & n_{y} \tau_{1 y} \\ 1 & 0 & 1 \\ 0 & 0 & 0 \\ 0 & n_{x} \tau_{1 x} & n_{x} \tau_{1 y} \\ 0 & 0 & 0\end{array}\right.$

$\left.\begin{array}{ccc}0 & 0 & 0 \\ n_{y} \tau_{1 x} & n_{y} \tau_{1 y} & 0 \\ 0 & 0 & 0 \\ \tau_{1 x}^{2} & 2 \tau_{1 x} \tau_{1 y} & \tau_{1 y}^{2} \\ n_{x} \tau_{1 x} & 2 n_{y} \tau_{1 x}+n_{x} \tau_{1 y} & 2 n_{y} \tau_{1 y} \\ 1 & 0 & 1\end{array}\right) \times\left(\begin{array}{c}{\left[\partial^{2} u / \partial x^{2}\right]} \\ {\left[\partial^{2} u / \partial x \partial y\right]} \\ {\left[\partial^{2} u / \partial y^{2}\right]} \\ {\left[\partial^{2} u / \partial x^{2}\right]} \\ {\left[\partial^{2} v / \partial x \partial y\right]} \\ {\left[\partial^{2} v / \partial y^{2}\right]}\end{array}\right)$

$=-\left(\begin{array}{cccc}\partial \tau_{1 x} / \partial \alpha^{1} & \partial \tau_{1 y} / \partial \alpha^{1} & 0 & 0 \\ 2 \partial n_{x} / \partial \alpha^{1} & \partial n_{y} / \partial \alpha^{1} & \partial n_{y} / \partial \alpha^{1} & 0 \\ 0 & 0 & 0 & 0 \\ 0 & 0 & \partial \tau_{1 x} / \partial \alpha^{1} & \partial \tau_{1 y} / \partial \alpha^{1} \\ 0 & \partial n_{x} / \partial \alpha^{1} & \partial n_{x} / \partial \alpha^{1} & 2^{\partial n_{y}} / \partial \alpha^{1} \\ 0 & 0 & 0 & 0\end{array}\right)\left(\left[\begin{array}{c}{\left[\partial y_{\partial x}\right]} \\ {\left[\partial y_{y y}\right]} \\ {\left[\partial y_{\partial x}\right]} \\ {\left[\partial y_{y}\right]}\end{array}\right)\right.$

$\left\{\begin{array}{r}\partial^{2} q_{x} / \partial \alpha^{1^{2}} \\ \partial R_{v x} / \partial \alpha^{1}\end{array}\right.$

$+\left(\begin{array}{c}\operatorname{Re}\left\{[\partial p / \partial x]+[\rho] /[\mu]^{\partial q_{x}} / \partial t\right\} \\ \partial^{2} q_{y} / \partial \alpha^{1^{2}} \\ \partial R_{v y} / \partial \alpha^{1} \\ \operatorname{Re}\left\{[\partial p / \partial y]+[\rho] /[\mu] \partial q_{y} / \partial t\right\}\end{array}\right)$

$\left(\begin{array}{ccc}\tau_{1 x}^{2} & 2 \tau_{1 x} \tau_{1 y} & \tau_{1 y}^{2} \\ n_{x} \tau_{1 x} & n_{x} \tau_{1 y}+n_{y} \tau_{1 x} & n_{y} \tau_{1 y} \\ 1 & 0 & 1\end{array}\right)\left(\begin{array}{c}{\left[\partial^{2} p / \partial x^{2}\right]} \\ {\left[\partial^{2} p / \partial x \partial y\right.} \\ {\left[\partial^{2} p / \partial y^{2}\right]}\end{array}\right)$

$=\left(\begin{array}{c}\partial^{2} R_{p} / \partial \alpha^{1^{2}} \\ \partial R_{p n} / \partial \alpha^{1} \\ R_{p l}\end{array}\right)-\left(\begin{array}{cc}\partial \tau_{1 x} / \partial \alpha^{1} & \partial \tau_{1 y} / \partial \alpha^{1} \\ \partial n_{x} / \partial \alpha^{1} & \partial n_{y} / \partial \alpha^{1} \\ 0 & 0\end{array}\right)$

$\left.\times\left(\begin{array}{l}{[\partial p / \partial x} \\ {[\partial p / \partial y}\end{array}\right]\right)$, where the jump condition for the scaled velocity (the augmented variables) is denoted by $\boldsymbol{q}=[\boldsymbol{v}]$, and the other quantities appearing on the right hand sides are defined by

$$
\begin{gathered}
R_{v x}=\operatorname{Re}\left(\frac{f_{n} n_{x}-f_{x}}{J}\right)-2\left(\frac{\partial q_{x}}{\partial \tau} \tau_{x}+\frac{\partial q_{y}}{\partial \tau} \tau_{y}\right) n_{x} \\
R_{v y}=\operatorname{Re}\left(\frac{f_{n} n_{y}-f_{y}}{J}\right)-2\left(\frac{\partial q_{x}}{\partial \tau} \tau_{x}+\frac{\partial q_{y}}{\partial \tau} \tau_{y}\right) n_{y} \\
R_{p}=\frac{f_{n}}{J}+\frac{2}{\operatorname{Re}}\left(\left[\frac{\partial u}{\partial x}\right] n_{x}^{2}+\left[\frac{\partial u}{\partial y}\right] n_{y} n_{x}\right. \\
\left.+\left[\frac{\partial v}{\partial x}\right] n_{x} n_{y}+\left[\frac{\partial v}{\partial y}\right] n_{y}^{2}\right)
\end{gathered}
$$

$$
R_{p n}=\frac{1}{J} \frac{\partial f_{\tau 1}}{\partial \alpha^{1}}+\frac{2}{\operatorname{Re}} \frac{\partial}{\partial \tau}\left(\left[\frac{\partial u}{\partial x}\right]\right) n_{x} \tau_{x}
$$$$
+\frac{2}{\operatorname{Re}} \frac{\partial}{\partial \tau}\left(\left[\frac{\partial u}{\partial y}\right]\right) n_{x} \tau_{y}
$$$$
+\frac{2}{\operatorname{Re}} \frac{\partial}{\partial \tau}\left(\left[\frac{\partial v}{\partial x}\right]\right) n_{y} \tau_{x}+\frac{2}{\operatorname{Re}} \frac{\partial}{\partial \tau}\left(\left[\frac{\partial v}{\partial y}\right]\right) n_{y} \tau_{y}
$$$$
-\frac{[\rho]}{[\mu]}\left(\frac{\partial q_{x}}{\partial t} n_{x}+\frac{\partial q_{y}}{\partial t} n_{y}\right)+\frac{2}{R e}\left[\frac{\partial u}{\partial x}\right] \frac{\partial}{\partial \tau}\left(n_{x} \tau_{x}\right)
$$$$
+\frac{1}{R e}\left[\frac{\partial u}{\partial y}\right] \frac{\partial}{\partial \tau}\left(n_{y} \tau_{x}+n_{x} \tau_{y}\right)
$$$$
+\frac{1}{R e}\left[\frac{\partial v}{\partial x}\right] \frac{\partial}{\partial \tau}\left(n_{x} \tau_{y}+n_{y} \tau_{x}\right)
$$$$
+\frac{2}{R e}\left[\frac{\partial v}{\partial y}\right] \frac{\partial}{\partial \tau}\left(n_{y} \tau_{y}\right),
$$

$$
R_{p l}=2\left[\frac{\rho}{\mu^{2}}\left(\frac{\partial u}{\partial x} \frac{\partial v}{\partial y}-\frac{\partial u}{\partial y} \frac{\partial v}{\partial x}\right)\right]
$$

$f_{n}=f_{x} n_{x}+f_{y} n_{y}$,

$f_{\tau 1}=\frac{f_{x} \tau_{x}+f_{y} \tau_{y}}{J}$.

[1] H. Miura et al., J. Plasma Phys. 72, 1095 (2006).

[2] H. Miura et al., Fusion Sci. and Technol. 51, 8 (2007).

[3] T-H. Watanabe et al., Nucl. Fusion 47, 1383 (2007).

[4] M. Sato and N. Nakajima, Phys. Plasmas 13, 102507 (2006).

[5] K. Kusano et al., Phys. Plasmas 5, 2582 (1998).

[6] Z. Li and K. Ito, The Immersed Interface Method, SIAM Frontiers in Applied Mathematics (2006) p. 20.

[7] S. Xu and Z. Jane Wang, SIAM J. Sci. Comput. 27, 1948 (2006).

[8] S. Xu and Z. Jane Wang, J. Comput. Phys. 216, 454 (2006).

[9] S. Xu and Z. Jane Wang, Comput. Methods Appl. Mech. 197, 2068 (2008), doj:10.10.16/j.cma.2007.06.012

[10] K. Ito and Z. Li, Appl. Math. Lett. 19, 229 (2006). 